\title{
A PRIORI EStimates fOR THE SOLUTION OF THE DIFFERENCE ANALOGUE OF A PARABOLIC DIFFERENTIAL EQUATION*
}

\author{
A. A. SAMARSRI I \\ (MOscov) \\ (Received 4 March, 1961)
}

When finite difference methods are used to solve a differential equation one of the most important theoretical problems which arises is to determine the convergence of the difference scheme in question when the difference net is divided up in an unestricted way.

The difference $z$ between the solution $y$ of the difference boundary problem and the solution $u$ of the corresponding problem for the original differential equation usually satisfies a non-homogeneous difference equation with homogeneous boundary and initial conditions. The right-hand side $\psi$ of this equation denotes the approximation error for the difference scheme on the solution $u$ of the original problem.

The question of the convergence of the difference scheme reduces to the estimate of the function $z$ in the following form.

$$
\|z\|_{1} \leqslant M\|\psi\|_{2}
$$

where \|\|$_{1}$ and \|\|$_{2}$ are norms and $M$ is a positive constant independent of the difference net.

Work devoted to a priori estimates for simple difference approximations of parabolic type differential equations has appeared recently [1], [2].

A particular interest is attached to a priori estimates when there are difference schemes for which the principle of the maximum does not, in general, hold good.

In the study of the convergence of difference schemes in the class of

- Zh. vych, at. 1, No. 3, 441-460, 1961. 
smooth coefficients, the asymptotic orders of approximation and accuracy usually coincide, i.e.

$$
\|z\|_{11} \leqslant M\|\psi\|_{1},
$$

where $\|z\|_{1}=\max _{\omega_{h}}|z|$ and $\omega_{h}$ is the difference net.

For discontinuous coefficients this is not generally true (see [3], [4], [5]). In the neighbourhood of a discontinuity of the coefficients the difference operator, generally speaking, does not approximate to the differential operator. Therefore we cannot apply the principle of the maximum in the study of convergence. Other a priori estimates with a specially selected norm must be found.

In the article [3], using the example of schemes for the very simple equation

$$
L^{(k, q, f)} u=\frac{d}{d x}\left[k(x) \frac{d u}{d x}\right]-q(x) u+f(x)=0
$$

it was shown that convergence in the class of discontinuous coefficients follows from the a priori estimate of the form (a), where

$$
\|\psi\|_{2}=\sum_{i=1}^{N-1} h\left|\sum_{k=1}^{i} h \psi_{k}\right| \text {. }
$$

An a priori estimate was obtained in [1] on the assumption that the difference analogue of the heat conduction coefficient was "differentiable" with respect to $x$.

In Section 1 we derive a similar a priori estimate free from this restriction, and this enables us to use the estimate for stationary (motionless) discontinuities of the heat conduction coefficient as well. We shall consider the difference boundary problem with boundary conditions of a very general form.

In Section 2 we obtain an integral formula enabling us to obtain $a$ priori estimates on the assumption only that the net functions, the coefficients of the equation, are bounded. These estimates are valid for the axisymmetric case and for the spherically-symmetric case. The new a priori estimates are an effective means for proving the convergence also of the estimate of the accuracy of the difference schemes in the class of discontinuous coefficients.

However we shall consider questions of convergence and accuracy of the various difference schemes separately. 


\section{First a priori estimate}

In this section we obtain an a priori estimate for a six-point difference equation on the assumption that the coefficients of the equation and of the boundary conditions are differentiable.

\section{Notation}

We give the necessary definitions and notation.

Let $\bar{\Pi}=(0 \leqslant x \leqslant 1,0 \leqslant t \leqslant T)$ be the basic region. We cover it with a difference net $\bar{\Omega}_{h \tau}$ with the nodal points $\left(x_{i}, t_{j}\right)$ where $x_{i}=i \cdot h, i=$ $0,1,2, \ldots, N, h=1 / N, t_{j} \geqslant j \cdot \tau, j=0,1,2, \ldots, L, r=T / L$. Let $\omega_{h}^{x}$ be the net with respect to $x$, i.e. the set of points $x_{i^{\prime}}=i^{\prime} \cdot h \cdot i^{\prime}=$ $0,1, \ldots, i=x / h$ and let $\omega_{\tau}^{t}$ be the set of points $t_{j}^{\prime}=j^{\prime}, \tau, j^{\prime}=0,1$, $\ldots, j=t / r$.

Then $\bar{\Omega}_{h \tau}=\omega_{h}^{1} \times \omega_{\tau}^{t}$. Let $\Omega_{h \tau}$ be the set of internal points $\left(x_{i}, t_{j}\right)$ of the region $\Omega_{h T}$, for which $0<i<N, 0<j<L$.

Let $z_{i}^{j}$ or $z\left(x_{i}, t_{j}\right)$ be some net function given on the net $\bar{\Omega}_{h r}$.

For the sake of simplification in writing we shall omit the indices $i$ and $j$ everywhere, and instead of $z_{i}^{j}$ we shall simply put $z$ or $z(x, t)$, not forgetting that here $(x, t)$ is an nodal point of the net, i.e. a point of the set $\bar{\Omega}_{h \tau}$. We shall also not indicate the dependence of the net functions on the net and shall write $z$ instead of $z^{h}$ or $z^{(h, \tau)}$.

For the difference ratios we use the following notation:

$$
\begin{aligned}
& z_{\bar{x}}=\frac{z(r, l)-z(x-h, t)}{h}, \quad z_{\lambda}=\frac{z(x+h, t)-z(x, t)}{h}, \\
& z_{\bar{l}}=\frac{z(x, t)-z(x, t-\tau)}{\tau}, \quad z_{t}=\frac{z(x, t+\tau)-z(x, l)}{\tau} .
\end{aligned}
$$

In addition, we shall write

$$
\begin{array}{rlrl}
\check{z} & =z(x, t-\tau), & \hat{z} & =z(x, t+\tau), \\
z^{(-1)} & =z(x-h, t), \quad z^{(+1)}=z(x+h, t) .
\end{array}
$$

The expression

$$
\frac{\Delta\left(a_{i}^{j} \nabla z_{i}^{j}\right)}{h^{2}}=\frac{a_{i+1}^{j}\left(z_{i+1}^{j}-z_{i}^{j}\right)-a_{i}^{i}\left(z_{i}^{j}-z_{i-1}^{j}\right)}{h^{2}} \quad\left(\Delta z_{i}=\nabla z_{i+1}=z_{i+1}-z_{i}\right)
$$

using this notation will be written in the form $\left(a z_{\bar{x}}\right) x_{x}$.

We shall be using various sums taken over the net $\omega_{h}^{1}$ or over part of it. We shall use an indexless notation for them too: 


\section{A.A. Samarskii}

$$
\begin{aligned}
& (\varphi, \psi)==\sum_{i=1}^{x-1} \varphi_{i} \psi_{i} h, \quad(\varphi, \psi]==\sum_{i=1}^{N} \varphi_{i} \psi_{i} h \\
& \lfloor\varphi, \psi\rangle=\sum_{i=0}^{N-1} \varphi, \psi, h, \quad[\varphi, \psi]=\sum_{i=0}^{N} \varphi_{i} \psi_{i} h \\
& \sum_{\omega_{h}^{i}} \psi \cdot h=\sum_{0 \leqslant W^{\prime}} h \psi\left(x^{\prime}\right)=\sum_{k \rightarrow 0}^{i} \psi_{k} \cdot h,
\end{aligned}
$$

where $\phi_{i}$ and $\psi_{i}$ are arbitrary functions given on the net $\omega_{h}^{1}$.

For a priori estimates we use the norms

$$
\begin{aligned}
& \|\psi\|_{0}=\max _{0 \leqslant i \leqslant N}\left|\psi_{2}\right|, \quad\|\psi\|_{1}=(|\psi|, 1), \\
& \|\psi\|_{2}==(\psi, \psi)^{12}, \quad\|\psi\|_{3}=\|\varphi\|_{2}, \\
& \varphi=\sum_{i^{\prime}=1}^{i} h \psi_{i^{\prime}},\|\psi\|_{4}-\|\psi\|_{3} \frac{1}{i} \mid(\psi, 1)_{i},\|\psi\|_{5}=-\left\|x^{-\left(m+-y^{\prime} / 2\right)} \varphi\right\|_{2}, \\
& 1<m<3 / 2,\|\psi\|_{6}=\|\ln (1 / x) \varphi\|_{2},\|\widetilde{\psi}\|_{\sigma}=\max _{1 \leqslant j^{\prime} \leqslant L}\left\|\psi^{j^{\prime}}\right\|_{\sigma}, \\
& \sigma=0,1,2,3,4,5,6 \text {, }
\end{aligned}
$$

It is clear that $\|\psi\|_{3} \leqslant\|\psi\|_{1} \leqslant\|\psi\|_{2} \leqslant\|\psi\|_{0}$.

\section{Green's difference formulae}

Let $\phi$ and $\psi$ be arbitrary net functions defined on the net $\omega_{h}^{(1)}$.

Using the identities

$$
\begin{gathered}
(\varphi \psi)_{x}=\varphi \psi_{x}+\psi^{(+1)} \varphi_{x}=\varphi^{(+1)} \psi_{x}+\psi \varphi_{x}, \\
\varphi\left(a \psi_{x}\right)_{x}=\left(a \varphi \psi_{x}\right)_{x}-a^{(+1)} \varphi_{x} \psi_{x},
\end{gathered}
$$

it is not difficult to show that the difference analogues of Green's formulae for the operator $L_{h} \psi=\left(a \psi_{\vec{x}}\right)$ are valid:

1) the first Green formula is

$$
\left(\varphi, L_{h} \psi\right)=-\left(a \psi_{\bar{x}}, \psi_{\bar{x}}\right]+a_{. N} \varphi_{N} \psi_{\bar{x}, .,}-a_{1} \varphi_{0} \psi_{x, 0}
$$

2) the second Green formula is

$$
\left(\varphi, L_{h} \psi\right)-\left(\psi, L_{h} \varphi\right)=a_{N}\left(\varphi \psi_{\bar{x}}-\psi \varphi_{\bar{x}}^{-}\right)_{N}-a_{1}\left(\varphi \psi_{x}-\psi \varphi_{\lambda}\right)_{k}
$$


We shall frequently use the first Green formula, and also the formulae for summation by parts:

$$
\begin{aligned}
& \left(\varphi, \psi_{\lambda}\right)=-\left(\psi, \psi_{\lambda}\right\rfloor-(\varphi \psi)_{\lambda}-\psi_{1} \psi_{0}, \\
& \left(\varphi, \psi_{\lambda}\right) \cdots\left(\psi, \varphi_{\lambda}\right)-\varphi_{\lambda} \psi_{\lambda},-(\varphi \psi)_{0} .
\end{aligned}
$$

\section{The simplest inequalities}

Lemma 1. For any function $\psi$ given on the net $\omega_{h}^{(1)}$, we have:

$$
\begin{aligned}
& \psi^{2}(x)-2 \psi_{i i}^{2}+2 \psi_{x}^{2}+1_{i 2}\left(\psi_{x}, \psi_{x}\right], \\
& \psi^{2}(x)<2 \psi_{1}^{2}+2\left(\psi_{x}, \psi_{x}\right) \text {. } \\
& \psi^{2}(x) \leqslant 2 \psi \div-2\left(\psi_{-}, \psi_{-}\right)
\end{aligned}
$$

To prove (a) for example, we need the identity

$$
\psi(x)=(1-x) \sum_{n} h \psi_{. x}\left(x^{\prime}\right)-x \sum_{x, x_{1}} h \psi_{x}\left(x^{\prime}\right)+(1-x) \psi_{0}+x \psi_{1}
$$

and the inequalities

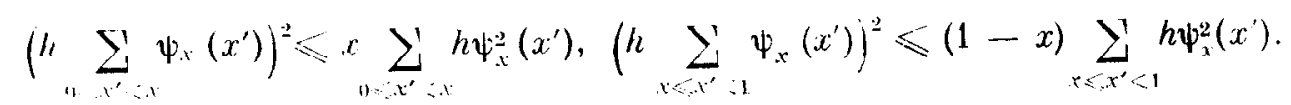

In particular, if $\psi_{0}=\psi_{N}=0$ then

$$
\psi^{2}(x) \leqslant i_{i}^{1}\left(\psi_{x}, \psi_{x}\right]
$$

Lemma 2. If the net function $\psi(x)$ given on $\omega_{h}^{(1)}$ satisfies the condition $\psi_{N}=0$ then

$$
\begin{aligned}
& \psi^{2}(x) \leqslant \frac{2 m--1}{m-1}\left(x+\frac{h}{2}\right)^{-(m-1)}\left(\left(x-\frac{h}{2}\right)^{m}, \psi_{x}^{2}\right], \quad 1<m<2, \quad 0 \leqslant x<1 \\
& \psi^{2}(x) \leqslant\left(2-\ln -\frac{1}{x+\frac{h}{2}}\right)\left(\left(x-\frac{h}{2}\right), \psi \frac{2}{x}\right], \quad m=1, \quad 0 \leqslant x<1 \\
& \| \psi \text { i }_{2}^{2} \leqslant \frac{2 m-1}{(2-m)(m-1)}\left(\left(x-\frac{h}{2}\right)^{m}, \quad \psi_{x}^{2}\right\rfloor, \quad 1<m<2 .
\end{aligned}
$$

For

$$
\begin{aligned}
\psi^{2}(x) & \leqslant\left(\sum_{x<x^{\prime}<1} h \psi_{x}\left(x^{\prime}\right) \cdot\left(x^{\prime}+\frac{h}{2}\right)^{m / 2} \cdot\left(x^{\prime}-\frac{h}{2}\right)^{-m / 2}\right)^{2} \leqslant \\
& \leqslant \sum_{x \leqslant x^{\prime}<1} h\left(x^{\prime}+\frac{h}{2}\right)^{m} \psi_{x}^{2}\left(x^{\prime}\right) \cdot \sum_{x \leqslant x^{\prime}<1}^{\prime} h\left(x^{\prime}+\frac{h}{2}\right)^{-m} \leqslant
\end{aligned}
$$




$$
\leqslant \frac{2 m-1}{m-1}\left(x+\frac{h}{2}\right)^{-(m-1)}\left(\left(x-\frac{h}{2}\right)^{m} \cdot \psi \cdot \frac{2}{x} j\right.
$$

since

$$
\sum_{x \leqslant x^{\prime}<1} h\left(e^{\prime}+\frac{h}{2}\right)^{-m} \leqslant \frac{2 m-1}{m-1}\left(x+\frac{h}{2}\right)^{-(m-1)}
$$

If $\psi_{N} \neq 0$, then the expressions on the right of the inequalities in the lemma must be multiplied by 2 , and $2 \psi_{N}^{2}$ must then be added to them.

To transform the product into a sum when constructing the upper bound we use the inequality (see [6])

$$
\prod_{k=1}^{r} x_{k}^{v} \leqslant \sum_{i k=1}^{r} v_{k} x_{k}, \text { where } x_{k} \geqslant 0, v_{k}>0, \sum_{k=1}^{r} v_{k}=1
$$

\section{The difference boundary problem}

In the basic region $\bar{\Omega}_{h r}=\omega_{h}^{(1)} \times \omega_{h}^{(T)}$ we consider the boundary problem for the equation which is the difference analogue of a parabolic differential equation

$$
\rho z_{\bar{t}}-\alpha\left(a z_{\bar{x}}\right)_{x}-(1-\alpha)\left(\check{a} z_{\bar{x}}\right)_{x}=-\Psi^{(x)} \text { on } \Omega_{h \tau},
$$

where $a$ is a numerical parameter, $0 \leqslant a \leqslant 1$

$$
\Psi^{(a)}=\alpha \Psi+(1-\alpha) \stackrel{\Psi}{\Psi}+\psi, \quad \Psi=(b z)_{\dot{x}}+c z_{\ddot{x}}+q z .
$$

The required function $z$, the coefficients $\rho, a, b, c, q$ and also $\psi$ are net functions given in the basic region $\bar{\Omega}_{h t}$ and, generally speaking, dependent on the steps $h$ and $r$ of the net. However, to simplify the writing we shall not indicate this dependence.

For $x=0(i=0)$ and $x=1(i=N)$ we have the boundary conditions

$$
\begin{aligned}
& \left(2 a^{i-1)} z_{x}+(1-\alpha) a^{2}(+1)^{2} z_{x}=\mathscr{E}_{1} z_{\bar{i}}+\alpha s_{1} z+(1-\alpha) \check{s}_{1}^{2} z_{,}, \quad x=0(i=(1),\right.
\end{aligned}
$$

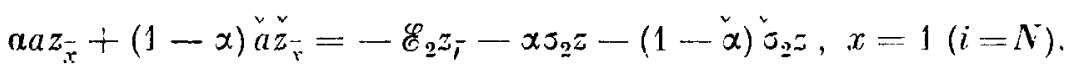

the coefficients $\mathscr{E}_{k}$ and $\sigma_{k}$ of which $(k=1,2)$ are functions given on the net $\omega_{\tau}^{(T)}$ and dependent, generally speaking, on $h$ and $r$.

We note that the boundary conditions of this kind are the difference analogue of the boundary condition for the differential equation

$$
\mathscr{E} \frac{\partial u}{\partial t}=a \frac{\partial u}{\partial x}--j u
$$


where $\mathscr{E}=\mathscr{E}(t), a-a(t), \sigma=\sigma(t)$ are functions of $t \in[0, T]$.

Difference boundary conditions of a higher order of accuracy, corresponding to the boundary conditions of the $3 \mathrm{rd}$ kind for the differential equation for heat conduction are of the same form as (8).

At the inilial moment of time, the function $z$ satisfies the condition

$$
z=0, \quad t=0(j=0) \text {. }
$$

The boundary condition of the first kind $z=0$, for $x=0$ for example, can be obtained formally from (8) if we put $a=1$ and take the limit as $\sigma_{1} \rightarrow \infty$ (assuming all the other coefficients to be bounded).

The linear equation (6) is obtained for the error $z=y-u$ ( $y$ is the solution of the difference problem, $u$ is the solution of the problem for the differential equation) not only for linear, but also for non-linear parabolic equations.

In this article, however, the equation (6) and the relation between its coefficients $a, b, \rho$ etc. on the coefficients of the original differential equation will not interest us.

\section{Conditions for the coefficients}

We assume everywhere that the coefficients of the difference boundary problem (6) - (9) satisfy the conditions:

$K_{1}$ ) the net runctions $\rho$ and $a$ are bounded below by a positive constant, independent of $h$ and $r$ :

$$
\rho \geqslant M_{0}>0, \quad a \geqslant M_{0} \rightleftharpoons 0
$$

$\mathrm{K}_{2}$ ) the net functions $b, c$ and $q$ are bounded in absolute magnitude by constants which are independent of the net (i.e. of $h$ and $r$ ):

$$
b\left|\leqslant b^{*}, \quad\right| q\left|\leqslant M_{1}, \quad\right| c \mid \leqslant c^{*}
$$

$\left.\mathrm{K}_{3}\right)$ all the coefficients $\mathscr{E}_{k}, \sigma_{k}(k=1,2)$ are non-negative;

$\left.K_{4}\right)$ at least one of the coefficients $\sigma_{k}(k=1,2)$ is bounded below by a positive constant $\sigma_{*}$ independent of $h$ and $r$.

It follows that there cannot occur cases when $\sigma_{1}=0$ and $\sigma_{2}=0$ simultaneously, and also that $\sigma_{1} \rightarrow 0$ and $\sigma_{2} \rightarrow 0$ as $h \rightarrow 0$ and $r \rightarrow 0$.

Any constant which is positive and not dependent on $h$ and $r$ we shall denote by $M$. We shall usually not indicate the structure of the constants $M$ nor their dependence on other constants. 
It is not difficult to see that the following lemma is true.

Lemma 1*. If the conditions $K_{1}, K_{3}$ and $K_{4}$ are satisfied, then for any net function $v$ given on the net $\omega_{h}^{(1)}$

$$
v^{2} \leqslant M I
$$

where

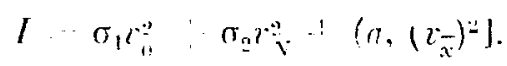

\section{The initial formula}

As we can see, the "differentiability" properties of the net functions $a(x, t), b(x, t)$ and $\rho(x, t)$ will play an important role in the estimate of the solution of problem (6) - (9).

In Section 7 we shall obtain an a priori estimate for $z$ with the norm II $\|_{0}$ on the assumption that the net function $a(x, t)$ satisfies the lipschitz condition with respect to $t$ in the region $\bar{\Omega}_{h r}$, and that the net function $b(x, t)$ satisfies the Lipschitz condition with respect to $x$; in other words, the difference ratios $a_{\bar{t}}$ and $b_{\bar{x}}$ are bounded:

$\mathrm{K}_{5}$ )

$$
\left|a_{-}\right| \leqslant M, \quad\left|b_{-}\right| \leqslant M \text {. }
$$

Let us pass now to the derivation of a basic identity which we shall use to obtain the a priori estimate

$$
\|z\|_{r}<M\|\Psi\|_{2} \text {. }
$$

We make a scalar multiplication of both sides of equation (6) by $r z \bar{t}$ :

$$
\tau\left(p, z_{\bar{t}}^{2}\right)-\alpha \tau\left(\left(a z_{\bar{x}}\right)_{x}, z_{\bar{t}}\right)-(1-\alpha) \tau\left(\left(a \check{z} \check{z}_{\bar{x}}\right)_{x}, z_{\bar{t}}\right)=\tau\left(\Psi^{(\alpha)}, z_{\bar{t}}\right) .
$$

To transform the second and third terms of the left-hand side we use Green's first difference formula:

$$
\begin{aligned}
& -\alpha \tau\left(\left(a z_{x}\right)_{x}, z_{t}\right)-(1-\alpha)\left(\left(a z_{x}\right)_{x}, z_{i}\right)-\alpha\left(a, z_{x}^{2}\right]-(1-\alpha)\left(a, z_{x}^{2}\right]
\end{aligned}
$$

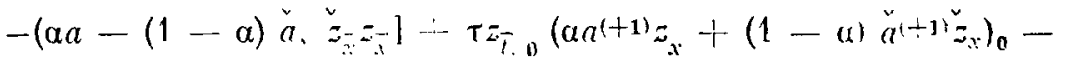

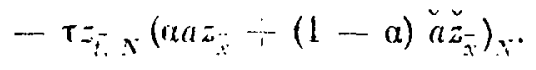

Then, using the boundary conditions (8) we find

$$
\begin{aligned}
& \tau \bar{\tau}_{i, 0}\left(\alpha a^{(+1)} z_{x}+(1-\alpha) \check{a}^{\left.(+1) \check{z}_{x}\right)_{0}=}\right. \\
& \quad=\tau \mathscr{E}_{1}\left(z_{\bar{\imath}, 0}\right)^{2}+\dot{+} \alpha \sigma_{1} z_{0}^{2}-(1-\alpha) \check{\sigma}_{1} z_{0}^{2}-\left(\alpha \sigma_{1}-(1-\alpha) \check{\sigma}_{1}\right) \check{z}_{0} z_{0},
\end{aligned}
$$




$$
\begin{aligned}
& -\tau z_{\bar{t}, N}\left(\alpha a z_{\bar{x}}+(1-\alpha) \dot{a} \check{z}_{\bar{x}}\right)_{N}= \\
& \quad=\tau \mathscr{E}_{2}\left(z_{\bar{t}, N}\right)^{2}+\alpha \sigma_{2} z_{N}^{2}-(1-\alpha) \check{\sigma}_{2}^{2} z_{N}^{2}-\left(\alpha \sigma_{2}-(1-\alpha) \check{\sigma}_{2}\right) \check{z}_{N} z_{N} .
\end{aligned}
$$

Putting these expressions in (11) and then in (10) we obtain the initial identity

$$
\tau\left[\rho, z_{\bar{t}}^{2}\right]-\alpha I=\tau\left(\Psi^{(\alpha)}, z_{\vec{t}}\right)+(1-\alpha) \grave{I}+Q
$$

where

$$
\begin{aligned}
& I=\left(a, z_{\tau}^{2}\right]+\sigma_{1} z_{0}^{2}+\sigma_{2} z_{N}^{2} \\
& {\left[p, z_{\bar{t}}^{2}\right]=\left(p, z_{\bar{t}}^{2}\right)+\mathscr{E}_{1}\left(z_{\bar{t}, n}\right)^{2}+\mathscr{E}_{Y}\left(\bar{z}_{\bar{i}, N}\right)^{2}} \\
& ?=\left(\alpha a-(1-\alpha) \check{a}, \check{z}_{\bar{x}} z_{\bar{x}}\right]+\left(\alpha \sigma_{1}-(1-\alpha) \check{\sigma}_{1}\right) \check{z}_{0} z_{0}+\left(\alpha \sigma_{2}-(1-\alpha) \check{\sigma}_{2}\right) \check{z}_{N^{\prime}} z_{\text {. }}
\end{aligned}
$$

For the first boundary problem, when we have the conditions $z=0$ for $x=0$ and $x=1$ instead of (8), relation (12) becomes

$$
\left(\rho, z_{\vec{\imath}}^{\ddot{\imath}}\right)+\alpha I=\tau\left(\Psi^{(\alpha)}, z_{\vec{\imath}}\right)+(1-\alpha) \check{I}+\left(\alpha a-(1-\alpha) \check{a}, \check{z}_{\bar{x}} z_{\vec{x}}\right]
$$

where

$$
I=\left(a, z_{x}^{2}\right]
$$

It can be obtained from (12) by putting $z_{0}=z_{N}=0$.

To evaluate the expression $Q$ in (12) we require the following lemma.

Lema? If the function $g(t) \geqslant 0$ given on the net satisfies the condision

$$
\left|g_{\bar{i}}\right| \leqslant M_{1} \sqrt{g g^{*}}
$$

then

$$
\begin{gathered}
|(\alpha g-(1-\alpha) \ddot{g}) v \grave{v}| \leqslant \\
\leqslant \frac{1}{2}\left(|2 \alpha-1|+(1-\alpha+|2 \alpha-1|) M_{1} \tau\right)\left(g v^{2}+\check{g} \check{v}^{2}\right),
\end{gathered}
$$

where $v$ is an arbitrary net function on $\omega_{\tau}^{(T)}$.

For, since $\sqrt{ } g \leqslant \sqrt{ } \mathscr{E}\left(1+M_{1} r\right)$, we can write

$$
\begin{aligned}
& |(\alpha g-(1-\alpha) \check{g}) v \check{v}|=|((1-\alpha)(g-\check{g})+(2 \alpha-1) g) v \check{v}| \leqslant \\
& \quad \leqslant(1-\alpha) M_{1} \tau V_{g \check{g}}^{\bar{g}}|v \check{v}|+|2 \alpha-1|\left(1+M_{1} \tau\right) \sqrt{g \check{g}}|v \check{v}|
\end{aligned}
$$


'This gives (16), since

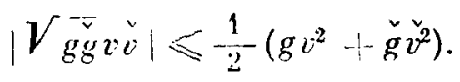

It is not difficult to see that the condition of Lenma 3 is satisfied for the function $a$ from conditions $K_{1}$ and $K_{5}$.

Let us make the requirement that $\sigma_{1}$ and $\sigma_{2}$ satisfy the condition (15):

$$
\left.\mathrm{K}_{6}\right)\left|\left(\sigma_{s}\right)_{1}\right| \leqslant M_{1} \sqrt{\sigma_{s} \bar{\sigma}_{s}}, \quad s=1,2 .
$$

Then, from Lemma 3, we can estimate the expression in formula (12):

$$
|Q| \leqslant-\frac{1}{2}\left(|2 \alpha-\cdots 1|+(1-\alpha+|2 \alpha-1|) M_{1} \tau\right)(I+\hat{I}) .
$$

This, together with the identity (12), gives us the inequality

$$
2 \tau\left|\rho, z_{i}^{2}\right|+\left(1-\alpha M_{1} \tau\right) I \leqslant\left(1+\alpha M_{1} \tau\right) I^{2}+2 \tau\left(\Psi^{(x)}, z_{\bar{l}}\right),
$$

if $0.5 \leqslant a \leqslant 1$.

Let us now transform the expression

where $\quad \Psi=(b)_{\bar{x}}+c z_{x}+q z$.

$$
\left(\Psi^{(x)}, z_{\bar{t}}\right)=u\left(\Psi, z_{-}^{-}\right)+(1-\alpha)\left(\check{\Psi}, z_{-}\right)+\left(\psi, z_{-}^{-}\right),
$$

Since the coefficient $b$ satisfies condition $K_{5}$, we can write

$$
\begin{aligned}
\left\{\left(\Psi^{\prime}, z_{\bar{t}}\right) \mid\right. & \left.\leqslant(\mid b(-1)+c) z_{\bar{x}}+\left|\left(q+b_{\bar{x}}\right) z\right|,\left|z_{\bar{t}}\right|\right) \leqslant \\
& \leqslant M\left\{\left(z_{\bar{x}}, z_{-}^{-}\right)+(z . z)\right\}+\frac{1}{4}\left(p, z_{\bar{l}}^{2}\right)
\end{aligned}
$$

or

$$
\left|\left(\Psi, z_{-}\right)\right| \leqslant M \cdot I+\frac{1}{4}\left(p,\left(z_{\vec{t}}\right)^{2}\right),
$$

using the conditions $K_{1}, K_{2}$ and the inequality

$$
|u v| \leqslant \frac{1}{4} A u^{2}+\frac{1}{A} v^{2}
$$

where $A>0$ is an arbitrary number, together with the Cauchy-Bunyakovskii inequality and condition $\mathrm{K}_{4}$, which gives

$$
(z, z) \leqslant M I \quad\left(\text { Lemma } 1^{*}\right)
$$

Since 


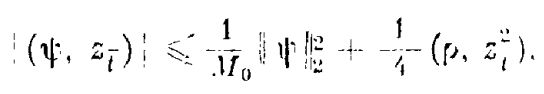

we can transform (18) to the form

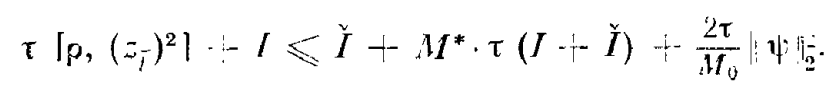

If $r$ is sufficiently small, so that $\tau<\tau_{n}$, it follows that

$$
I \leqslant x \check{I}+\frac{2}{M_{0}} x \cdot \tau \| \psi \mu_{2}, \text { where } x=\frac{1+M^{*} \tau}{1-M^{*} \tau}
$$

7. An a priori estimate

We solve inequality $(20)$ with the initial condition

$$
I^{0}=0 \text { when } t=0
$$

Successively applying inequality (20) we find

or

$$
I^{j} \leqslant \frac{2}{M_{0}} \sum_{j^{\prime}=1}^{j} \tau x^{j-j^{\prime}+1}\left\|\psi^{j^{\prime}}\right\|_{2}^{2} \leqslant M \sum_{j^{\prime}=1}^{j} \tau\left\|\psi^{j^{\prime}}\right\|_{2}^{2},
$$

$$
I^{j} \leqslant M\left\|_{1} \widetilde{\psi}\right\|_{2}^{2}, \quad \text { if } \quad \tau<\tau_{0}
$$

From Lemma $1^{*}$ we conclude that

$$
\left\|z^{j}\right\|_{0} \leqslant M\left[\sum_{j^{\prime}=1}^{j} \tau\left\|\psi^{j^{\prime}}\right\|_{2}^{2}\right]^{1 / z}, \text { or } \widetilde{\|z\|_{0}} \leqslant M\|\widetilde{\psi}\|_{2} \text {. }
$$

We have thus proved the following theorem.

Theorem 1. The solution of the difference boundary problem (6) - (9) has, for sufficiently small $r \leqslant r_{0}$ the a priori estimate

$$
\|z\|_{0} \leqslant M\left[\sum_{j^{\prime}=1}^{j} \tau\left\|\psi^{j^{\prime}}\right\|_{2}^{2}\right]^{1 / 2}, 0,5 \leqslant \alpha \leqslant 1
$$

or

$$
\widetilde{\| z}\left\|_{10} \leqslant M \widetilde{\|}\right\|_{2}
$$

if conditions $K_{1}$ to $K_{6}$ are satisfied. 
It is clear from the above reasoning that the theorem is also true for the first boundary problem. The proof in this case will be simpler. It will be necessary to put $z_{0}=z_{N}=0$ formally throughout.

\section{Notes}

1. If the original function $\left.z\right|_{t=0}=\phi(x) \neq 0$, then, as it is not difficult to show, the a priori estimate

$$
\|z\|_{0} \leqslant M\left\{\left(\sum_{j^{\prime}=1}^{j} \tau \| \psi^{j^{\prime} \|_{2}^{2}}\right)^{1 / 2}+\left(I^{0}\right)^{1 / 2}\right\} \text {, where } I^{0}=\sigma_{1} \varphi_{0}^{2}+\sigma_{2} \varphi_{N}^{2}+\left(a(x, 0),\left(\varphi_{\bar{x}}\right)^{2}\right]
$$

will be valid.

2. The condition $\left|a_{t}\right| \leqslant 4\left(K_{5}\right)$ does not exclude the possibility of there being stationary discontinuities of the net function $a(x, t)$ as $h \rightarrow 0$, i.e. discontinuities for fixed $x=$ const for all $t \in \omega_{\tau}{ }^{T}$. The $a$ priori estimate (22) was obtained in [1], [2] for the case $a=1$ in the assumption that $\left|a_{x}\right| \leqslant M$, which excludes the case of stationary discontinuities in the heat conduction coefficient. If $a$ has a discontinuity on some line $x=\eta(t)\left(\eta_{t} \not \equiv 0\right)$ then the condition $\mathrm{K}_{5}$ cannot be satisfied. We shall call a discontinuity of this sort oblique or mobile. When there is an oblique discontinuity we need more exact estimates with the sole condition: $0<M_{1} \leqslant a \leqslant M_{2}$ for the coefficient a. Section 2 is devoted to this problem.

3. If homogeneous boundary conditions

$$
\begin{gathered}
\alpha a^{(+1)} z_{x}+(1-\alpha) \check{a}^{(+1)} \check{z}_{x}=\mathscr{E}_{1} z_{\bar{t}}+\alpha \sigma_{1} z+(1-\alpha) \check{\sigma}_{1} \check{z}-\mu_{1}, x=0(i=0), \\
\alpha a z_{\bar{x}}+(1-\alpha) \grave{a} z_{\bar{x}}=-\check{\mathscr{E}}_{2} z_{\bar{t}}-\alpha \sigma_{2} z-(1-\alpha) \check{\sigma}_{2} \check{z}-\mu_{2}, \quad x=1(i=N),
\end{gathered}
$$

are given for $x=0$ and $x=1$, then Theorem 1 still applies if, instead of $\left\|y_{2}\right\|_{2}^{2}$, we write the expression

$$
\overline{\| \psi_{2}^{2}}=\|\psi\|_{2}^{2}+\frac{\mu_{1}^{2}}{\mathscr{E}_{1}}+\frac{\mu_{2}^{2}}{\mathscr{E}_{2}},
$$

so that

$$
\|z\|_{2}^{2} \leqslant M \sum_{j^{\prime}=1}^{j} \tau \overline{\left\|\psi^{j^{\prime}}\right\|_{2}^{2}}
$$

4. Let us consider the more general differential equation

$$
p \frac{\partial u}{\partial t}=\frac{1}{r^{n-1}} \frac{\partial}{\partial r}\left(r^{n-1} k(r, t) \frac{\partial u}{\partial r}\right)+\frac{1}{r^{n-1}} \frac{\partial}{\partial r}\left(b(r, t) r^{n-1} u\right)+q u(r, t)+f .
$$


For $n=1$ we obtain a homogeneous equation, the value $n=2$ corresponds to the case of axial symmetry, and $n=3$ to the case of spherical symetry. Introducing the mass variable $x \sim r^{n}$, we can rewrite the equation in the form

$$
\begin{aligned}
& \rho \frac{\partial u}{\partial t}=\frac{\partial}{\partial x}\left(x^{m} k \frac{\partial u}{\partial x}\right)+\frac{\partial}{\partial x}\left(x^{m} b u\right)+q u+f \\
& \text { where } m=\frac{2(n-1)}{n}, 0 \leqslant m \leqslant \frac{4}{3} .
\end{aligned}
$$

The difference analogue of an equation of this kind is

$$
p z_{\bar{\imath}}-\alpha\left(A z_{\bar{x}}\right) x-(1-\alpha)\left(\check{A} \check{z}_{\bar{x}}\right)_{x}=\Psi^{(\alpha)},
$$

where

$$
\begin{gathered}
\Psi^{(\alpha)}=\alpha \Psi+(1-\alpha) \check{\Psi}+\psi, \quad \Psi=(B z)_{\bar{x}}+q z, \\
A=\left(x-\frac{h}{2}\right)^{m} a, \quad B=\left(x+\frac{h}{2}\right)^{m} \cdot b .
\end{gathered}
$$

The coefficient $a$ in the boundary conditions (8) must be replaced by $A$.

If a satisfies condition $K_{6}$, then $A$ also satisfies this condition.

Therefore all the reasoning which led to the inequality

$$
I(t) \leqslant M \sum_{j^{\prime}=1}^{j} \tau\left\|\psi^{j^{\prime}}\right\|_{2}^{2},
$$

still holds. $I(t)$ will be expressed by the formula

$$
I=\sigma_{1} z_{0}^{2}+\sigma_{2} z_{N}^{2}+\left(A, z_{x}^{2}\right]
$$

Using Lemma 2, we can write

$$
\|z\|_{2}^{2} \leqslant M I, \quad 0 \leqslant m<2,
$$

and we obtain the estimate

$$
\|z\|_{2}^{2} \leqslant M \sum_{j^{\prime}=1}^{j} \tau\left\|\psi^{j^{\prime}}\right\|_{2}^{2}, 1 \leqslant m<2 .
$$

In addition, we have

$$
z^{2} \leqslant M\left(x+\frac{h}{2}\right)^{-(m-1)} I, \quad m \neq 1 .
$$

If $m=1$, then we have 


$$
z^{i} \leqslant M \ln \frac{1}{x+\frac{h}{2}} \cdot I
$$

and, therefore, we obtain the a priori estimates:

1) if $m>1$ then

$$
\begin{gathered}
\|z\|_{0}^{2} \leqslant M h^{-(m-1)}\|\tilde{\psi}\|_{2}^{2}, \\
\left(|z|^{p}, 1\right)^{\frac{2}{p}} \leqslant M\|\psi\|_{2}, \text { where } 2 \leqslant p<\frac{2}{m-1} ;
\end{gathered}
$$

2) if $m=1$, then

$$
\left(|z|^{p} ; 1\right)^{1 / p} \leqslant \Delta M_{p}\|\widetilde{\psi}\|_{2}
$$

where $p=2$ is any number.

These estimates can possibly be improved. An a priori estimate similar to (22) also holds for the solution of a parabolic differential equation and for a foth scheme and the straight line method.

\section{The second a priori estimate}

In this section we obtain a priori estimates for the case of a fourpoint difference equation $(a=1)$ without assuming that the coefficients $a$ and $b$ are "differentiable".

\section{The boundary problem}

We consider the difference boundary problem (4) - (9) of Section 1 for a four-point equation $(a=1)$ :

$$
\begin{aligned}
& \rho z_{i}-(a z-)_{x}+q \cdot z=(b z)_{x}+c \cdot z_{x}+\psi \\
& a^{(+1)} z_{x}=\mathscr{E}_{1} z_{\bar{l}}+\sigma_{1} z, x=0, \quad t \in \omega_{\div}^{T} ; \\
& -a z_{\bar{x}}=\mathscr{E}_{2} z_{\bar{\imath}}+\sigma_{2} z, x=1, t \in \omega_{\tau}^{T} ; \\
& \left.z\right|_{t=0}=0, x \in \omega_{h}^{1} \text {. }
\end{aligned}
$$

We shall assume that the functions $\rho, a, q, b$ and $c$ are defined on the net $\Omega_{h \tau}$, and the functions $\mathscr{E}_{k}$ and $\sigma_{k}(k=1,2)$ are defined on the net $\omega_{\tau}^{(T)}$, and satisfy the conditions $K_{1}-K_{4}$ and, moreover, we shall make the requirement that the conditions

$\left.K_{i}\right)\left|\mathscr{E}_{k,-}\right| \leqslant M_{1} \cdot \mathscr{E}_{k}, l_{i}=1,2$, 
$\left.K_{8}\right)\left|p_{t}\right| \leqslant M_{1}$

are satisfied.

We introduce the new function $\vec{z}$, putting

$$
z=\bar{z}(1+\bar{M} \cdot \tau)^{\frac{t}{\tau}}\left(t \in \omega_{\tau}^{T}\right)
$$

where $\bar{M}$ is an arbitrary positive constant.

For the function $\bar{z}$ we obtain the following boundary problem:

$$
\begin{aligned}
& \rho \bar{z}_{\bar{t}}-\left(\overline{a z}_{\bar{x}}\right)_{x}+\bar{q} \bar{z}=\left(\overline{b z} \bar{x}_{\bar{x}}+\bar{c} \cdot \bar{z}_{\bar{x}}+\bar{\psi},\right. \\
& a^{(+1)} \bar{z}_{x}=\mathscr{E}_{1} \bar{z}_{\bar{t}}+\bar{\sigma}_{1} \bar{z}_{z}, \quad x=0 \\
& -\overline{a z}_{\bar{x}}=\mathscr{E}_{2} \bar{z}_{\bar{t}}+\bar{\sigma}_{2} \bar{z}, \quad x=1 \\
& \left.z\right|_{t=0}=0,
\end{aligned}
$$

where

$$
\begin{gathered}
\bar{a}=a(1+M \cdot \tau), \bar{b}=b(1+\bar{M} \cdot \tau), \bar{c}=c(1+\bar{M} \cdot \tau), \\
\bar{\sigma}_{m}=\sigma_{m}(1+\bar{M} \cdot \tau)+\bar{M} \cdot \mathscr{E}_{m}(m=1,2), \bar{q}=p \bar{M}+q(1+\bar{M} \cdot \tau), \\
\bar{\psi}=\psi /(1+\bar{M} \cdot \tau)^{\frac{1}{\bar{F}}-1} .
\end{gathered}
$$

We shall make our choice of the constant $\bar{M}$ more precise later (in Section 5). Meanwhile we assume that the choice of $\bar{M}$ ensures that the condition

$$
\tilde{q} \geqslant Q=\bar{M} M_{\mathrm{n}}+q(1+\bar{M} \cdot \tau)>0 .
$$

is satisfied. It is clear from this that without loss of generality we can take $q \geqslant 0$.

To simplify the writing we shall use the notation of the problem (1) (3), bearing in mind that we are in fact considering the problem $(5)-(7)$. llowever, for the coefficient of $z$ we retain for the time being the notation $\tilde{q}$.

In formulating the final results we shall turn to the initial problem (1) - (3) and take the transformation (4) into account.

2. The equation for the function $\mathrm{z}^{2 n}$

Let us consider the sequence of functions

$$
z=z . \stackrel{1}{z}=z^{2}, \ldots,{ }_{z}^{n}=\left(\begin{array}{c}
n-1 \\
z
\end{array}\right)^{2}, \ldots(n=0,1,2, \ldots),
$$


where $\stackrel{n}{z}=z^{2^{n}}$, and let us form the difference equation and difference boundary conditions which the function $z^{n}$ must satisfy if $z$ is a solution of the problem (1) - (3).

We use the obvious identities

$$
2 z \cdot z_{\bar{x}}=\left(z^{2}\right)_{\bar{x}}+h\left(z_{\bar{x}}\right)^{2}, 2 z \cdot z_{x}=\left(z^{2}\right)_{x}-h\left(z_{x}\right)^{2}, 2 z \cdot z_{\bar{t}}=\left(z^{2}\right)_{\bar{t}}+\tau\left(z_{\bar{t}}\right)^{2}
$$

and write the recurrence relations

$$
\left(\begin{array}{c}
n \\
a z_{\bar{x}}
\end{array}\right)_{x}=2 \stackrel{n-1}{z}\left(\begin{array}{c}
n-1 \\
a z_{\bar{x}}
\end{array}\right)_{x}+a^{(+1)}\left(\begin{array}{c}
n-1 \\
z_{x}
\end{array}\right)^{2}+a\left(\begin{array}{c}
n-1 \\
z_{\bar{x}}
\end{array}\right)^{2}, \quad z_{\bar{t}}^{n}=2^{n-1} z_{\bar{t}}^{n-1}-\tau\left(\begin{array}{c}
n-1 \\
z_{\bar{t}}
\end{array}\right)^{2}
$$

From this we find

$$
\begin{gathered}
\left(\begin{array}{c}
n \\
a z_{\bar{x}}
\end{array}\right)_{x}=2^{n} \cdot z^{2^{n}-1}\left(a z_{\bar{x}}\right)_{x}+\sum_{k=0}^{n-1} 2^{n-k-1}\left[a\left(\begin{array}{l}
k \\
z_{\bar{x}}
\end{array}\right)^{2}+a^{(+1)}\left(\begin{array}{l}
k \\
z_{x}
\end{array}\right)^{2}\right] \cdot z^{2^{n}-2^{k+1}} \\
n \\
z_{\bar{t}}=2^{n} \cdot z^{2^{n}-1} z_{\bar{t}}-\tau \sum_{k=0}^{n-1} 2^{n-k-1}\left(\begin{array}{l}
k \\
z_{\bar{t}}
\end{array}\right)^{2} z^{2^{n}-2^{k+1}} .
\end{gathered}
$$

For ${\stackrel{n}{z_{x}}}_{x}$ and ${ }^{n} z_{x}$ we obtain expressions similar to (10) (in the case of ${ }_{z}^{n}$ the sign + stands in front of the sum).

We have used the relation

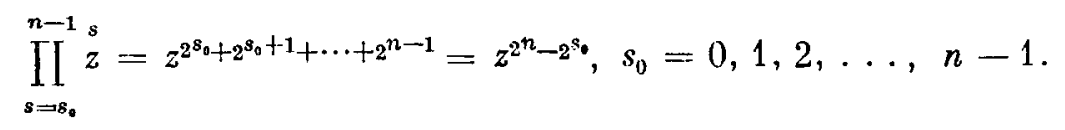

As a result, we obtain the following boundary problem for the function

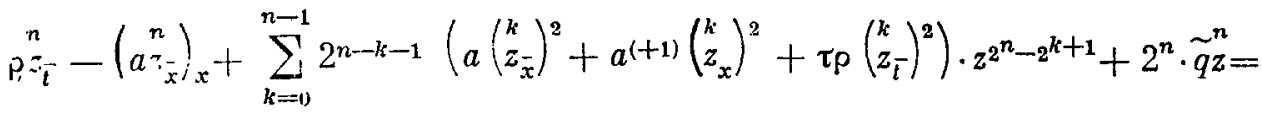

$$
\begin{aligned}
& =2^{n} z^{2 n-1} \Psi, \Psi=(b z)_{\bar{x}}+c z_{\bar{x}}+\psi ; \\
& \left.\left.a^{(-1)} z_{x}^{n}=\mathscr{E}_{1} z_{\bar{\imath}}^{n}+2^{n} \sigma_{1} z+\sum_{k=0}^{n-1} 2^{n-k-1}(h a(+1))^{k} z_{x}\right)^{2}+\tau \mathscr{E}_{1}\left(\begin{array}{l}
k \\
z_{\bar{i}}
\end{array}\right)^{2}\right) \cdot z^{2^{n}-2^{k+1}}, x=0, \\
& -a_{\bar{x}}^{n}=\mathscr{E}_{2} z_{\bar{\imath}}^{n}+2^{n} \sigma_{2} z+\sum_{k=0}^{n-1} 2^{n-k-1}\left(h a\left(\begin{array}{l}
k \\
z_{\bar{x}}
\end{array}\right)^{2}+\tau \mathscr{E}_{2}\left(\begin{array}{l}
k \\
z_{\bar{t}}
\end{array}\right)^{2}\right) \cdot z^{2^{n-2} 2^{k+1}}, x=1 ; \\
& \left.z\right|_{t=0} ^{n}=0 \text {. }
\end{aligned}
$$


It is seen that, because of conditions $K_{1}$ and $K_{3}$

$$
\begin{gathered}
\max _{\omega_{\tau}^{t}} z_{0} \leqslant \max _{\omega_{\tau}^{t}} z_{1}, \\
\max _{\omega_{\tau}^{t}} z_{\mathrm{V}} \leqslant \max _{\omega_{\tau}^{t}}^{n} z_{N-1} .
\end{gathered}
$$

Therefore it will be sufficient to obtain the estimate for the function $z_{i}$ at internal points of the net $\omega_{h}^{(1)}$.

\section{Integral formulae of the nth rank}

To derive the basic integral relation for the function $\stackrel{n}{z}$ we make $a_{n}$ scalar multiplication of equation (11) by 1 and calculate the sum $\left(\left(a z-\bar{z}^{-} x^{\prime}\right.\right.$ 1). To do this, we make use of Green's first formula and the boundary conditions (12):

$$
\begin{aligned}
& -\left(\left(\begin{array}{c}
n \\
z_{\bar{x}}
\end{array}\right)_{x}, 1\right)=-\left(\begin{array}{c}
n \\
z_{\bar{x}}
\end{array}\right)_{N}+a_{1}^{n} z_{x, 0}^{n}=\mathscr{E}_{1} z_{\overline{t, 0}}^{n}-\mathscr{E}_{2}^{n} z_{\bar{t}, N}+2^{n}\left(\begin{array}{c}
n \\
\sigma_{1} z_{0}+\sigma_{2} z_{N}
\end{array}\right)+ \\
& +\cdot \sum_{k=0}^{n-1} 2^{n-k-1}\left\{\left(h a_{1}\left(\begin{array}{l}
k \\
z_{x, 0}
\end{array}\right)^{2}-\tau \mathscr{E}_{1}\left(\begin{array}{l}
k \\
z_{\bar{t}, 0}
\end{array}\right)^{2}\right) z_{0}^{2^{n-2^{k+1}}-1}\right. \\
& +\left(h a_{N}\left(\begin{array}{l}
k \\
z_{\bar{x} . N}
\end{array}\right)^{2}+\tau \mathscr{E}_{2}\left(\begin{array}{l}
k \\
z_{\bar{i}, N}
\end{array}\right)^{2}\right) z_{N}^{\left.2^{n-2} 2^{k+1}\right\}}
\end{aligned}
$$

Then, since

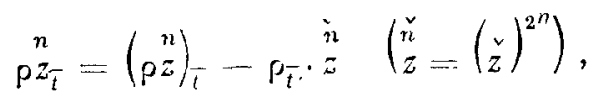

using the notation

$$
\begin{aligned}
& {\left[\begin{array}{l}
n \\
\rho,
\end{array}\right]=\left(\begin{array}{l}
n \\
\rho, z
\end{array}\right)+\mathscr{E}_{1} z_{0}+\mathscr{E}_{2} z_{N}\left(\rho_{0}=\frac{\mathscr{\varepsilon}_{1}}{h}, \rho_{N}-\frac{\mathscr{E}_{2}}{h}\right)} \\
& {\left[\begin{array}{ll}
n & n \\
q & z
\end{array}\right]=(\tilde{q}, \quad z)+\sigma_{1} z_{0}+\sigma_{2} z_{N},}
\end{aligned}
$$

we obtain the following identity:

$$
\begin{aligned}
& {\left[\rho, z_{\bar{t}}+2\left(a,\left(\begin{array}{c}
n-1 \\
z_{\bar{x}}
\end{array}\right)^{2}\right]+\sum_{k=0}^{n-2} 2^{n-k-1}\left\{\left(a\left(\begin{array}{c}
k \\
z_{\bar{x}}
\end{array}\right)^{2}, z^{2^{n}-2^{k+1}}\right]+\left[a^{(+1+1)}\left(\begin{array}{l}
k \\
z_{x}
\end{array}\right)^{2}, z^{z^{n}-2^{k}+1}\right)\right\}+\right.} \\
& +2^{n}\left[\tilde{q}, \begin{array}{l}
n \\
z
\end{array}\right]+\tau \sum_{k=0}^{n-1} 2^{n-k-1}\left[P\left(\begin{array}{c}
k \\
z_{\bar{t}}
\end{array}\right)^{2}, z^{2^{n}-2^{k+1}}\right]=2^{n}\left(z^{2^{2}-1}, \Psi\right)+\left[P_{\bar{t}}, \stackrel{\check{n}}{z}\right],
\end{aligned}
$$


which we shall call an integral identity of the $n$th rank $(n \geqslant 1)$.

then $n=1$ we obtain the identity of the first rank:

$$
\text { [p. } \left.z^{2}\right]_{\bar{t}}+2\left(a,\left(z_{\bar{x}}\right)^{2}\right]+2\left[\tilde{a} \cdot z^{2}\right]+\tau\left[\rho \cdot\left(z_{-}\right)^{2}\right]=-2(z, \Psi)+\left[\rho_{-} \cdot \check{z}^{2}\right]
$$

which the solution of the boundary problem (1) - (3) must satisfy.

By leaving out the underlined expression on the left-hand side of the identity, we obtain the integral inequality of the nth rank

$$
[\rho, z]_{\bar{t}}+2^{n-1} I+2^{n}\left[Q, \begin{array}{l}
n \\
z
\end{array}\right] \leqslant 2^{n}\left(z^{2^{n}-1}, \Psi\right)+\left[P_{\bar{t}}, \begin{array}{l}
n \\
z
\end{array}\right] \text {, }
$$

where

$$
\stackrel{n-1}{I}^{\prime}=\left(a,\left(\begin{array}{c}
n-1 \\
z_{\bar{x}}
\end{array}\right)^{2}\right]+\sum_{k=0}^{n-2} 2^{n-k-2}\left\{\left(a\left(\begin{array}{l}
k \\
z_{\bar{x}}
\end{array}\right)^{2}, z^{2^{n}-2^{k-1}}\right]+\left[a^{(+1)}\left(\begin{array}{l}
k \\
z_{x}
\end{array}\right)^{2}, z^{2^{n}-2^{k+1}}\right)\right\} .
$$

We make use of this inequality when constructing a priori estimates of the solution of the problem (1) - (3).

\section{Estimates for the right-hand side}

Consider first of all the expression

$$
2^{n}\left(z^{\nu}, \Psi\right), \text { where } \Psi=(b z)_{\bar{x}}+c z_{\bar{x}}+\psi, v_{n}=2^{n}-1 .
$$

Without loss of generality we can take $b=0$ for $x=0$ and $x=x_{N-1}$.

For, let us put $b=\tilde{b}+f$ where $f$ is a linear function equal to $b$ for $x=0$ and $x=x_{N-1}$ so that $\widetilde{b}_{0}=\widetilde{b}_{N-1}=0$. Then we shall have

$$
(b z)_{\bar{x}}+c z_{\bar{x}}=(\hat{b} z)_{\bar{x}}+\left(c+f^{(-1)}\right) z_{\bar{x}}+f_{\bar{x}} \cdot z, f_{\bar{x}}=\frac{b_{N-1}-b_{0}}{1-h} .
$$

The summand $2^{n}\left(f_{\bar{x}^{z}} z, z^{\nu}\right)=2^{n}\left(f_{\bar{x}^{\prime}}, z^{\nu}\right)$ can be combined with the term $2^{n}(Q, \stackrel{n}{z})$ on the left. Then we obtain the expression

$$
Q=\bar{M} \cdot M_{0}+q(1+\bar{M} \cdot \tau)-f_{\bar{x}}>0,
$$

for $Q$, where $\bar{M}$ is an arbitrary constant which we shall choose later.

To simplify the writing, we shall as before write $b$ instead of $\tilde{b}$ and $c$ instead of ${ }^{\prime} \widetilde{c}=c+f^{(-1)}$.

Since $b_{0}=b_{N-1}=0$, from formula (4) of Section 1 we have 


$$
\left(z^{2} n,(b z)_{x}\right)=-\left(\left(z^{v}\right)_{x}, b z\right)
$$

We derive a formula for the difference ratio

$$
\left(z^{v} n\right)_{x}:=\left(z^{(+1)}\right)^{v_{n-1}} \cdot \cdot^{n-1} z_{x}+{ }^{n-1} \cdot\left(z^{v_{n-1}}\right)_{x} .
$$

Successively using this recurrence relation, we find

$$
\left(z^{v_{n}}\right)_{x}==\sum_{k=0}^{n-1}\left(z^{(+1)}\right)^{v} k \cdot z^{v n^{-v} k+1} \cdot z_{x}^{k} .
$$

We put (19) in (18) and use the inequality

$$
\stackrel{n}{z} \leqslant M \stackrel{n-1}{(\text { Lemma } 1 *)}
$$

together with the Hölder inequality:

$$
\begin{aligned}
& \left|2^{n} \sum_{k=0}^{n-1}\left(\left(z^{+1}\right)^{v_{k}} \cdot z^{v_{n}-v_{k+1}}{ }_{z_{x}}^{k}, b z\right)\right| \leqslant \\
& \leqslant 2^{n} \cdot M \sum_{k=0}^{n-1} \mid(1, z)^{n / 2^{n-k}-1 / 2^{n}} \cdot\left(1, z^{n}\right)^{1 / 2-1 / 2^{n-k}+1 / 2^{n}} \cdot\left(z^{v_{n}-v_{k+1}},\left(\begin{array}{l}
k \\
z_{x}
\end{array}\right)^{2}\right)^{1 / 2} \leqslant \\
& \leqslant M \cdot 2^{n}\left(1, \begin{array}{l}
n \\
z
\end{array}\right)^{1 / 2} \cdot \sum_{k=0}^{n-1}\left(z^{v_{n}-a_{+1}}, a^{(+1)}\left(\begin{array}{l}
k \\
z_{x}
\end{array}\right)^{2}\right)^{1 / 3}\left(b_{N-1}=0\right) .
\end{aligned}
$$

Therefore, since

$$
\sum_{k=0}^{n-1}\left|v_{k}\right|^{1 / 2} \leqslant \sqrt{n}\left(\sum_{k=0}^{n-1}\left|v_{k}\right|\right)^{1 / 2}
$$

and using (17) for ${ }^{n-1}$ we obtain

$$
2^{n}\left|\left(z^{v} n,(b z)_{\bar{x}}\right)\right| \leqslant\left(M \cdot 2^{n} \cdot \sqrt{n}\right)^{2}(1, z)+\frac{1}{4}{ }^{n-1} I
$$

We turn now to the expression $\left(z^{n}, \psi\right)$. We introduce the function $\Phi$ defining it by the conditions

so that

$$
\Phi_{\bar{x}}=\psi, \Phi_{\bullet}=0
$$

$$
\Phi=\sum_{0<x^{\prime}<x} h \cdot \Psi\left(x^{\prime}\right),: \quad \Phi_{N}=\Phi_{N-1} \bullet
$$


Using the identity

$$
\left(z^{\nu}, \psi\right)=-\left(\left(z^{v}\right)_{x}, \Phi\right)+z_{N}^{v_{n}^{n}} \cdot \Phi_{N}
$$

by analogy with what we did before we find that

and therefore

$$
2^{n}\left|\left(z^{v_{n}}, \psi\right)\right| \leqslant M\left({ }^{n-1}\right)^{1-1 / 2^{n}} \cdot 2^{n}\left(\sqrt{n}\|\psi\|_{3}+\left|\Phi_{N}\right|\right)
$$

$$
2^{n}\left|\left(z^{v}, \psi\right)\right| \leqslant \frac{1^{2}}{4} I^{n-1}+\left(M \cdot 2^{n} \cdot \sqrt{n}\right)^{2^{n}}\left(\|\psi\|_{Q}\right)^{2^{n}} .
$$

The last summand $2^{n}\left(z^{\nu}, c z_{\bar{x}}\right)$ can be estimated very simply:

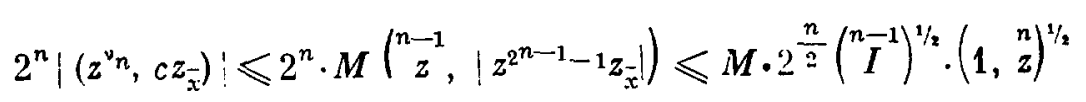

or

$$
2^{n}\left|\left(z^{v n}, c z_{\bar{x}}\right)\right| \leqslant M \cdot 2^{n}(1, \stackrel{n}{z})+\frac{1}{2}{ }^{n-1}
$$

since

$$
\left(a^{(+1)},{z^{2}-2}^{n-2}\left(z_{x}\right)^{2}\right) \leqslant{\frac{1}{2^{n-1}}}^{n-1}{ }^{\prime}
$$

Collecting together the estimates (20), (23) and (24) we obtain

$$
2^{n}\left|\left(z^{v_{n}}, \Psi\right)\right| \leqslant I^{n-1}+2^{n} \cdot M_{n}^{(2)}\left(1, \begin{array}{l}
n \\
z
\end{array}\right)+M_{n}^{(1)}\left(\|\Psi\|_{4}\right)^{2^{n}},
$$

where

$$
M_{n}^{(1)}=\left(M 2^{n} \cdot \sqrt{n}\right)^{2^{n}}, M_{n}^{(2)}=M \cdot n \cdot 2^{n},
$$

From conditions $K_{1}, K_{7}$ and $K_{8}$ we have

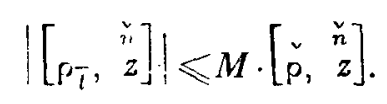

\section{A priori estimates}

Let us turn now to the inequality (16) and use estimates (25) and (27):

$$
[\rho, z]_{i}+\stackrel{n-1}{I}+2^{n}\left[Q, \begin{array}{l}
z \\
z
\end{array}\right] \leqslant M\left[\begin{array}{ll}
\dot{n} \\
\ddot{n}, & z
\end{array}\right]+2^{n} M_{n}^{(2)}\left(1, \begin{array}{l}
z \\
z
\end{array}\right)+M_{n}^{(1)}\left(\|\psi\|_{1}\right)^{2 n} .
$$

Let us now choose the arbitrary constant $\bar{M}$ so that 


$$
Q=\bar{M} M_{0}+(q-f)(1+\bar{M} \cdot \tau) \geqslant M_{n}^{(2)}\left(1+\bar{M}_{-}\right) .
$$

This condition will be satisfied if $r \leqslant r_{0}(n)$ where

$$
\tau_{0}(n) \leqslant \frac{M b^{*}}{n \cdot 2^{n}}+M
$$

It is clear from this that $r_{0}$ does not depend on $n$ when $b \equiv 0$.

When $M_{n}$ is chosen in this way, the inequality (28) can be rewritten in the form

$$
[\rho, \quad z]_{\bar{i}}+\stackrel{n-1}{I} \leqslant M\left[\begin{array}{ll}
\dot{p} & n \\
\dot{p} & z
\end{array}\right]+M_{n}^{(1)}\left(\|\psi\|_{4}\right)^{2^{n}}
$$

or

$$
\left[p, \quad \begin{array}{l}
z \\
]
\end{array}\right]+\tau \stackrel{n-1}{I} \leqslant(1+M \cdot \tau)\left[\begin{array}{ll}
\check{p}, & \check{z}
\end{array}\right]+\tau M_{n}^{(1)}\left(\|\psi\|_{4}\right)^{2^{n}}
$$

This gives us

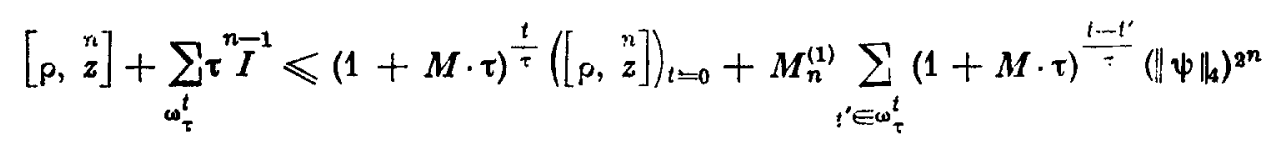

\section{Since}

$$
\stackrel{n}{z}=0 \text { for } t=0
$$

we obtain

or

$$
\sum_{\omega_{\tau}^{t}} \tau^{n-1} I \leqslant\left[p, \quad \begin{array}{l}
n \\
I
\end{array}\right] \leqslant M_{n}^{(1)} \frac{e^{M \cdot t}}{M} \sum_{\omega_{\tau}^{t}} \tau\left(\|\psi\|_{4}\right)^{2^{n}} \quad\left(\psi^{0}=0\right)
$$

$$
\|z\|_{1} \leqslant M \cdot M M_{n}^{(1)} \sum_{\omega^{l}:} \tau\left(\|\psi\|_{4}\right)^{2^{n}}
$$

This proves the following theorem.

Theorem 2. If conditions $K_{1}-K_{4}, K_{7}$ and $K_{8}$ are satisfied, then the estimates

$$
\left[\rho, z^{2^{n}}\right]^{\frac{1}{2^{n}}} \leqslant M_{n}\left[\sum_{j^{\prime}=1}^{j} \tau\left(\left\|\psi^{j}\right\|_{4}\right)^{2^{n}}\right]^{\frac{1}{2^{n}}} \leqslant M \cdot M_{n}\|\widetilde{\psi}\|_{4}
$$




$$
\left\|z^{2^{n}} \frac{1}{2_{1}^{n}} \leqslant M_{n}\right\| \|_{4}
$$

are valid for the solution of the difference boundary problem (1) - (3) for sufficiently small values of $\tau \leqslant \tau_{0}$, where $/ A_{n}=C_{1} \cdot 2^{n} \sqrt{n} e^{C_{2} \cdot 2 n \cdot n}$ and $n \geqslant 1$ is any integer, $C_{1} \cdot C_{2}$ are positive constants not depending on $n, h$ or $r$, and

$$
\tau_{0}=\tau_{0}(n) \leqslant M\left(1+\frac{b^{*}}{n \cdot 2^{n}}\right)
$$

The magnitude of $\tau_{0}$ does not depend on $n$ when $b=0$ or if $(b z)_{\bar{x}}$ is taken on the preceding layer $t-r$.

Let us derive one more a priori estimate. The inequality (31) gives

$$
\sum_{\omega_{\tau}^{t}} \tau{ }^{n-1} I^{-1} \leqslant M \cdot M_{n}^{(1)} \sum_{t i} \tau \tau\left(\|\psi\|_{4}\right)^{2^{n}}
$$

Then, since ${ }^{n} \leqslant M^{n} \bar{I}^{-1}$ (Lemma $1^{*}$ ), we have

$$
\sum_{\omega_{?}^{l}} \tau \cdot z \leqslant M \sum_{\omega_{\tau}^{t}} \tau \stackrel{n-1}{I}
$$

The following theorem follows from this and (35).

Theorem 3. If the conditions of Theorem 2 are satisfied, then we have the a priori estimate

$$
\left(\sum_{\omega_{\tau}^{t}} z^{2^{n}} \cdot \tau\right)^{12^{n}} \leqslant M M_{n}\|\widetilde{\psi}\|_{4}
$$

where $n \geqslant 1$ is any integer, $M_{n}$ is the constant of Theorem 2 and $M$ is a positive constant independent of $n, h$ and $r$.

To illustrate the effectiveness of the a priori estimate (34) let us consider the following example.

Let $\psi_{i}=1 / h\left(\delta_{i, i+1}-\delta_{i, i_{0}}\right)$ where $\delta_{i, k}$ is the Kronecker symbol, $0<i<\stackrel{i}{N}, 0<i_{\theta}<\stackrel{i}{N}-1$. Then $\|\psi\|_{2}=\sqrt{2} 2 / \sqrt{h}$; but

$$
\|\psi\|_{3}=\left\|\sum_{i^{\prime}=1}^{2} h \psi_{i^{\prime}}\right\|_{i^{2}}=\left\|\delta_{i, i_{0}}\right\|_{2}=\cdots \bar{h},\|\psi\|_{4}=\|\psi\|_{3}=\sqrt{h}
$$

and therefore 


$$
\left(\sum_{\omega_{h /}^{1}} z^{n} \cdot h\right)^{1} \leqslant M_{\nu} \cdot \sqrt{h}, \text { where } "=2^{n} .
$$

This gives us

$$
\|\widetilde{z}\|_{0} \leqslant M_{p} \cdot h^{12-1, n}
$$

Fixing $p=p_{0}>2(n>1)$ we obtain on the right a quantity which tends to zero as $h \rightarrow 0$.

Choosing $n$ dependent on $h$, it is not difficult to obtain the estimate

$$
\|z\|_{0} \leqslant M h^{1 / z-\rho(h)} \quad(b=0),
$$

for sufficiently small values of $h$, where $M$ is a constant which is independent of $h$ and $\tau$, and where $\rho(h) \rightarrow 0$ as $h \rightarrow 0$.

\section{Notes}

1. The a priori estimate (34) is also valid for the solution of a boundary problem for a differential equation the analogue of which is the difference boundary problem we have been considering. Of course, it must be remembered that

$$
\begin{gathered}
\left.\|z\|_{1}^{n}\right|^{1 / 2^{n}}=\left(\int_{0}^{1} z^{2^{n}} d x\right)^{1 / 2^{n}}, \\
\|\Psi\|_{4}=\left[\int_{0}^{1} d x\left(\int_{0}^{x} \psi d \xi\right)^{2}\right]^{1 / 2}+\left|\int_{0}^{1} \psi d x\right| .
\end{gathered}
$$

2. Theorems 2 and 3 are valid for the solution of the same boundary problem for the differential-difference equation of Roth [5]

$$
p z_{\bar{t}}-\frac{d}{d x}\left[a(x, t) \frac{d z}{d x}\right]+q(x, t) z=\frac{d}{d x}(b(x, t) z)+c \frac{d z}{d x}+\psi
$$

in the region $0 \leqslant x \leqslant 1, t \in \omega_{\tau}^{(T)}$, where $\|z\|_{1}$ and $\|\psi\|_{4}$ are given by formulae (39) and (40).

3. Theorem 2 can be generalized for the boundary problem

$$
\begin{aligned}
& \rho z_{\bar{\imath}}-\left(A z_{\bar{\imath}}\right)_{x}+q \cdot z=(B z)_{\bar{x}}+\dot{\psi} ; \\
& A=a \cdot\left(x-\frac{h}{2}\right)^{m}, B=b \cdot\left(x+\frac{h}{2}\right)^{m}, \quad 0 \leqslant m<\frac{3}{2} ; \\
& A^{(+1)} z_{x}=\mathscr{E}_{1} z_{\bar{t}}+\sigma_{1} z, \quad x=0 ;-A z_{\bar{x}}=\mathscr{E}_{2} z_{\bar{t}}+\sigma_{2} z, \quad x=1 ; \\
& \left.z\right|_{t=0}=0,
\end{aligned}
$$


where $a, b, q, \rho, \mathscr{E}_{k}$ and $\sigma_{k}(k=1,2)$ satisfy the conditions of Theorem 2 . This equation is the difference analogue of the differential equation

$$
p \frac{\partial z}{\partial t}-\frac{\partial}{\partial x}\left(x^{m} a \frac{\partial z}{\partial x}\right)+q z=\frac{\partial}{\partial x}\left(x^{m} \cdot b z\right)+\psi_{3}
$$

which includes, as we showed in Section 1, Paragraph 8, the cases of axial $(n=1)$ and spherical $(m=4 / 3)$ symmetry.

The norm $\|\psi\|_{5}$ or $\|\psi\|_{6}$ (for $m=1$ ) is used for the estimates of the right hand side of $\psi$. The a priori estimate (34) takes the form

$$
\left\|z^{2^{n}}\right\|_{1}^{\frac{1}{2^{n}}} \leqslant M_{n}\|\widetilde{\Psi}\|_{\sigma}, \sigma=5 \text { or } 6 .
$$

4. If the original function $z=\phi(x) \neq 0$, then the a priori estimate for $z$ takes the form

$$
\left\|z^{2^{n}}\right\|_{1}^{\frac{1}{2^{n}}} \leqslant M_{n}\left[\|\widetilde{\Psi}\|_{4}+\|\varphi\|_{2}\right]+\left\|\varphi^{2^{n}}\right\|_{1}^{\frac{1}{2^{n}}}
$$

The case of a non-uniform net presents particular interest. We shall therefore discuss it separately.

\section{Non-uniform nets}

So far we have only been considering uniform nets with steps $h=1 / N$ and $r=T / L$. It is not difficult to show that the a prior $i$ estimates of Section 1 and Section 2 still hold for the non-uniform nets

$$
\begin{aligned}
\omega_{h}^{1} & =\left\{x_{0}=0, x_{1}, \ldots, x_{i}, \ldots, x_{N}=1, h_{i}=x_{i}-x_{i-1}\right\}, \\
\omega_{\tau}^{T} & =\left\{t_{0}=0, t_{1}, \ldots, t_{j}, \ldots, t_{L}=T, \tau_{j}=t_{j}-t_{j-1}\right\}
\end{aligned}
$$

with variable steps $h_{i}$ and $r_{j}$.

Let $z_{i}^{j}$ or $z\left(x_{i}, t_{j}\right)$ be any net function.

We use the following notation:

$$
\begin{aligned}
& z_{x, i}^{j}=\frac{z_{i}^{j}-z_{i-1}^{j}}{h_{i}}, \quad z_{x, j}^{j}=\frac{z_{i+1}^{j}-z_{i}^{j}}{h_{i+1}}, \\
& z_{\widetilde{x}, i}^{j}=\frac{z_{i+1}^{j}-z_{i}^{j}}{\hbar_{i}} \text { where } h_{i}=0,5\left(h_{i}+h_{i+1}\right), \\
& z_{\frac{i}{i, i}}^{j}=\frac{z_{i}^{j}-z_{i}^{j-1}}{\tau_{j}} .
\end{aligned}
$$

For convenience in writing we shall, as before, omit the indices $i$ and $j$ in the net function and simply write $z$ instead of $z_{i}^{j}$, putting 


$$
z^{(+1)}=z_{i+1}^{j}, \quad \check{z}=z_{i}^{j-1}, \quad \hat{z}=z_{i}^{j+1}, \quad z^{(-1)}=z_{i-1}^{j}, \quad h=h_{i}, \quad h^{(+1)}=h_{i+1} \text { etc. }
$$

so that

$$
z_{\bar{x}}=\frac{z-z^{(-1)}}{h}, \quad z_{x}=\frac{z^{(+1)}-z}{h^{(+1)}}=\left(z_{\bar{x}}\right)^{(+1)} \text { etc. }
$$

In this case there are two kinds of sum:

$$
\begin{aligned}
\text { 1) }(\varphi, \psi) & =\sum_{i=1}^{N-1} \varphi_{i} \psi_{i} h_{i},[\varphi, \psi)=\sum_{i=0}^{N-1} \varphi_{i} \psi_{i} h_{i+1}, \quad(\varphi, \psi]=\sum_{i=1}^{N} \varphi_{i} \psi_{i} h_{i}, \\
\text { 2) }(\varphi, \psi)^{*} & =\sum_{i=1}^{N-1} \varphi_{i} \psi_{i} \cdot \hbar_{i},
\end{aligned}
$$

where $\phi_{i}$ and $\psi_{i}$ are arbitrary net functions.

We introduce the following norms:

$$
\begin{gathered}
\|\psi\|_{0}=\max _{\omega_{h}^{1}}\left|\psi_{i}\right|,\|\psi\|_{1}=(1,|\psi|)^{*}, \\
\|\psi\|_{2}^{2}=(\psi, \psi)^{*},\|\psi\|_{3}=\|\Phi\|_{2}, \Phi=\Phi_{i}=\sum_{k=1}^{i} \psi_{h} \hbar_{k}, \\
\|\psi\|_{4}=\|\psi\|_{3}+\|\psi\|_{1} .
\end{gathered}
$$

The difference operator $L_{h} \psi$, which is equal to $\left(a \psi_{\bar{x}}\right)_{x}$ on a uniform net, is defined in the form $L_{h^{\prime}} L^{\prime}=\left(a \psi_{\bar{x}}\right) \tilde{x}_{\tilde{x}}$ on a non-uniform net, i.e.

$$
L_{h} \psi_{i}=\frac{1}{\hbar_{i}} \Delta\left(a_{i} \frac{\nabla \psi_{i}}{h_{i}}\right) ; \Delta \psi_{i}=\nabla \psi_{i+1}=\psi_{i+1}-\psi_{i} .
$$

Green's first formula on a non-uniform net becomes

$$
\left(\varphi,\left(a \psi_{\bar{x}}\right)_{\widetilde{x}}\right)^{*}=-\left(a, \varphi_{\vec{x}} \psi_{\bar{x}}\right]+\left(a \varphi \psi_{\vec{x}}\right)_{N}-\left(a^{(+1)} \varphi \psi_{x}\right)_{0} .
$$

Clearly the difference equation takes the form

$$
\rho z_{\bar{t}}-\alpha\left(a z_{\bar{x}}\right)_{\tilde{x}}-(1-\alpha)\left(\check{a} z_{\bar{x}}\right)_{\tilde{x}}=\Psi .
$$

An additional requirement must be introduced in the formulation of the theorems: the ratio $h_{i+1} / h_{i}$ of two neighbouring steps of the difference net is bounded on both sides:

$$
0<C_{1} \leqslant \frac{{ }^{h_{i+1}-1}}{h_{i}} \leqslant C_{2}
$$


where $C_{1}$ and $C_{2}$ are constants not depending on the number $N$.

This ratio, $h_{i+1} / h_{i}$, is a local characteristic of the non-uniformity of the net.

The basic inequality (22) of Theorem 1 takes the form

$$
\left\|z^{j}\right\|_{0} \leqslant M\left[\sum_{j^{\prime}=1}^{j} \tau_{j^{\prime}}, \psi^{j^{\prime}} \mid j^{2}\right]^{1 / 2} .
$$

The second a priori estimate is unchanged of course, though, the symbols in (34) must be understood to apply to the non-uniform net.

The use of the a priori estimates of Section 1 and Section 2 in the question of the convergence and accuracy of difference schemes for parabolic type equations will be considered separately.

In conclusion, the author is glad of the opportunity to express his gratitude to A.N. Tikhonov for his interest in this work, and also to I.V. Fryazinov, discussions with whom enabled several of the estimates to be made more exact.

\section{REFERENCES}

1. Lees, M., Energy inequalities for the solution of differential equations. Trans. Amer. Math. Soc., 94, 58-73, 1960.

2. Lees, M., Approximate solutions of parabolic equations. J. Soc. Industr. and Appl. Math., 7, 167-183, 1959.

3. Tikhonor, A.N. and Samarsk11, A.A., Zh. vychisl. mat. i mat. fiz., 1: $5-63,1961$.

4. Samarski1, A.A., Trud. Vses. soveshchaniya po differentsial'nym uravneniyam (Proceedings of the All-Union Conference on Differential Equations). (Yerevan, 10-17 November, 1958). Izd-vo Akad. Nauk Ara. S.S.R, 148-160, 1960 .

5. Samarski1, A. A., Nauchn. dokl, vyssh, shkoly. Fiz.-mat, nauki, No. 1, 48-53, 1959.

6. Hardy, G.H., Littlewood, D. E. and Polja, G., Nyeravenstra (Inequa 1ities). Izd-vo in. 11t., Moscor, 20, 1948. 